\title{
Gender Incidence in the Salary of Informal Workers for the Management of Socioeco- nomic Strategies
}

\section{Incidencia del Género en el Salario de Traba- jadores Informales Para la Gestión de Estrate- gias Socioeconómicas}

VII International Congress of

Science, Technology,

Entrepreneurship and

Innovation (SECTEI 2020)

Corresponding Author:

E. Melendres Medina

emmelendres@hotmail.com

Published: 26 August 2021

Production and Hosting by

Knowledge E

(c) E. Melendres Medina et al. This article is distributed under the terms of the Creative Commons Attribution License, which permits unrestricted use and redistribution provided that the original author and source are credited.

\section{E. Melendres Medina ${ }^{1}$, M. Campaña Lara ${ }^{2}$, B. Riera Riera ${ }^{3}$, and J. Orozco Carrillo ${ }^{4}$}

${ }^{1}$ Facultad de Administración de empresas, Escuela Superior Politécnica de Chimborazo, Extensión Macas, Riobamba, Ecuador

${ }^{2}$ Dirección de Investigación, Instituto Superior Tecnológico Stanford, Instituto Superior Tecnológico José Ortega y Gasset, Riobamba, Ecuador

${ }^{3}$ Dpto. de Planificación, Escuela Superior Politécnica de Chimborazo, Analista de Planificación Operativa

${ }^{4}$ Dpto. Administrativo, Escuela Superior Politécnica de Chimborazo, Director Administrativo, Riobamba, Ecuador

\section{Abstract}

This research examined the problems experienced by women who work in the informal sector of the city of Riobamba due to receiving poor and lower incomes than men. The main objective was to analyze the influence that gender has on wages of women in this sector, to establish strategies that can promote more equitable development. Qualitative and quantitative approaches were used using inductive and deductive methods. Data were collected through a survey and analyzed using the Chi square test. The results showed that women's wages were dependent on their gender, conditioned by a sociocultural environment that has not evolved, especially in the informal sectors made up mostly of rural population. Also, the presence of child labor was evident, and wages could not cover the basic needs of women who identified as heads of household with family responsibilities of between three to five people. The strategy identified to promote equity in the recognition and value of fair work without distinction of gender was based on the following elements: the implementation of inclusion policies and control, training of young women in non-traditional trades, promotion of changes in traditional patterns in households, and policies to combat poverty with a gender focus for sustainable social development.

Keywords: gender, equity, salary, strategies, socioeconomic, management.

\section{Resumen}

La investigación plantea la problemática que viven las mujeres que trabajan en el sector informal de la ciudad de Riobamba debido a su condición de género para percibir ingresos deficientes e inferiores a los hombres, el objetivo principal es analizar la incidencia que tiene el género en el salario de las mujeres de este sector para el establecimiento de estrategias que promuevan un desarrollo más equitativo. La metodología investigativa considera estudios analíticos, correlacionales, exploratorios, de campo, un enfoque cuali- cuantitativo, con métodos inductivos y deductivos, usando técnicas como la encuesta, considerado la relación

How to cite this article: Medina EM, Lara MC, Riera BR, Carrillo JO (2021). Gender Incidence in the Salary of Informal Workers for the Management of 
de dependencia a través del Chi cuadrado para la comprobación de la hipótesis, como resultados principales se puede encontrar que la incidencia del género en los salarios de las mujeres es dependiente, condicionado por un ambiente sociocultural que no ha evolucionado sobre todo en los sectores informales conformados en su mayoría por población rural, se evidencia la presencia del trabajo infantil y salarios que no pueden cubrir las necesidades básicas de mujeres que se identifican como jefes de hogar con cargas familiares de entre 3 a 5 personas, se plantea como solución la estrategia para promover la equidad en el reconocimiento y puesta en valor del trabajo equitativo sin distinción de género basada en 5 elementos: la implementación de políticas de inclusión y control, la capacitación de las jóvenes en oficios no tradicionales, promoción de cambios de patrones tradicionales en los hogares, políticas de combate a la pobreza con enfoque de género para un desarrollo social sustentable.

Palabras Clave: género, equidad, salario, estrategias, socioeconómicas, gestión.

\section{Introducción}

La Organización de las Naciones Unidas (ONU) (2015) en los Objetivos de Desarrollo Sostenible, agenda 2030, establece como una de las metas en las que todos los países deberían luchar, Lograr la igualdad entre los géneros y empoderar a todas las mujeres y las niñas (objetivo 5), evidenciando entre otros datos que:

Las mujeres en el norte de África acceden a menos de uno de cada cinco empleos remunerados en el sector no agrícola. La proporción de mujeres en empleos remunerados fuera del sector agrícola ha aumentado del 35\% en 1990 al $41 \%$ en 2015. En 46 países, las mujeres ahora ocupan más del 30\% de los escaños en el parlamento nacional en al menos una cámara (p. 2). [1]

Cada país tiene marcados sus datos en cuanto a la desigualdad a la que ha sido expuesta la mujer hasta la presente fecha, éste es el tema que el estudio pone en discusión para conocer la realidad en Ecuador.

La Organización Internacional del Trabajo (OIT) [2] sugiere que el desarrollo social y económico de los pueblos debe basarse en principios que promuevan la equidad y propendan a disminuir las brechas.

Según datos del Instituto Nacional de Estadísticas y Censos (INEC) [3], la tasa global de empleo durante los años 2007 a 2019 encuentra una cifra significativa de 95,4\% para el caso de las mujeres, y en caso de los hombres de 96,7\%, evidenciándose una tasa inferior en 1,3 puntos para el género femenino, en el área del empleo adecuado la diferencia es mucho más significativa considerándose 14,2 puntos porcentuales por debajo del género masculino, así a nivel nacional el $44,8 \%$ de varones cuenta con un empleo pleno y el de la mujer se ubica en el 30,6\%; La tasa de subempleo para 
los hombres es 2,8 puntos porcentuales mayor que de las mujeres, en el caso del desempleo la tasa para los hombres es 1,3 puntos porcentuales menor que de las mujeres, la diferencia también se ve reflejada en el sueldo que perciben las mujeres llegando a $\$ 292,7$ en promedio y el de los varones $\$ 345,5$ esto si consideramos solo el sector formal de la economía.

De acuerdo a datos del Plan de desarrollo cantonal de Riobamba (2014-2019) podemos conocer que la provincia de Chimborazo, presenta un alto nivel de migración del sector rural al urbano incrementando el trabajo informal especialmente de mujeres y niñas abriendo aún más las brechas de pobreza, en un desarraigo cultural y de la familia que ha modificando profundamente el mercado laboral informa el Instituto de Economía Popular y Solidaria en su diagnóstico 2014 de la zona 3. Cabe indicar que culturalmente para la mujer el trabajo en el campo (agricultura), no es considerado como parte de una actividad productiva remunerada sino como una responsabilidad de las actividades domésticas [4].

Una de las condiciones que le permite a mujer superar las barreras socio económicas es la independencia financiera o al menos el contar con un ingreso monetario como producto de su actividad productiva, sin embargo, al ingresar en el mercado laboral el trato es diferenciado, de alguna manera los estudios en Ecuador evidencias las diferencias salariales en los sectores formales de la economía, pero ¿Qué pasa con el sector informal? donde se observa una mayor cantidad de mujeres ejerciendo actividades de comercio si salimos a los mercados de nuestras ciudades [5]. A simple vista las diferencias son mucho más marcadas y el trabajo pretende evidenciar una realidad aparente que permita que el tema del salario de la mujer del sector informal sea tomado en cuenta en los planes de desarrollo de las economías locales.

El problema central que el estudio plantea son los deficientes ingresos que perciben las mujeres como fruto de su trabajo en el sector informal de la ciudad de Riobamba, una brecha que a pesar del paso del tiempo no ha sido superada social y económicamente y parece profundizarse convirtiéndose en una barrera para el desarrollo de los pueblos.

Ante esta problemática el estudio se plantea como objetivo analizar la incidencia que tiene el género en el salario de las mujeres que trabajan en el sector informal de la ciudad de Riobamba para el desarrollo de estrategias socio económicas efectivas.

\section{Materiales y Métodos}

\subsection{Género en el sector productivo}

La educación de género es necesaria ubicarla en un contexto socio cultural, este entorno, ejerce influencia en el acontecer y la vivencia nacional, en la que el estado 
tiene que establecer políticas que nos lleven a gozar de una sociedad más equitativa, sin distinción de género, ni clases sociales, raza o religión [6].

La identidad de género da al hombre y a la mujer, características que se las asumen como naturales en nuestra sociedad [7]. Estas características aprendidas y aceptadas responden a un tiempo y patrón cultural determinado; lamentablemente cada sociedad ha tenido como representante exclusivo al hombre, el rol de la mujer ha sido de estar a expensas o beneficiaria de la labor realizada por él, y aun cuando ella haya tenido una participación protagónica, esta es minimizada y hasta olvidada. La inequidad de género es más visible en los jóvenes y adultos, cuando se empieza a disminuir la capacidad de la mujer dentro de la sociedad, años han tenido que pasar para que la mujer tenga su representatividad en la sociedad y en cargos públicos.

Para Castaño citado por Rodríguez y Limas (2017)

Las propuestas principales para revisar la determinación de los salarios, el valor (no) asignado al trabajo y sus efectos sobre hombres y mujeres incluyen: (i) la Economía de la familia y la división del trabajo por género; (ii) Las diferencias salariales por género: El capital humano y la discriminación en el mercado de trabajo; (iii) El dualismo y segmentación del trabajo por género; (iv) La segregación ocupacional por género; (v) La economía marxista y el género; (vi) El género y la economía según las feministas; (vii) La crisis económica y el género; (viii) Las mujeres y la recesión; (ix) Los estudios económicos con perspectiva de género y $(x)$ La explotación de las fuentes estadísticas (p. 127) [8].

En términos económicos lo que las puede llevar a esta autonomía es el salario. Sin embargo, para acceder a él, deben entrar en un mercado laboral que, en determinados segmentos, tiene un trato diferenciado para algunos grupos sociales. Por ejemplo, una de las vías a través de las cuales esto es posible es por medio de salarios diferentes por igual trabajo entre hombres y mujeres. A ello se suma la tensión provocada por la carga de trabajo no remunerado, que muchas ocasiones se encuentra bajo exclusiva responsabilidad de las mujeres. La alta segregación ocupacional, tanto vertical como horizontal, hace que las mujeres no ocupen los mismos puestos de trabajo ni accedan a los niveles superiores de las ocupaciones a la par con los hombres, por último, otra de las causas que provoca la desigual participación laboral femenina y masculina son las características socioeconómicas de ellas; es decir, su nivel de educación, su nivel de ingreso, el acceso a servicios de cuidado, entre otros que, en muchas ocasiones, se encuentran vinculados a la carga total de trabajo.

Para Castillo y Salas (2018) para el período 2000-2017:

Se apreció que la distribución de los empleados es mayor en los hombres en cuatro categorías de ocupación: Jornalero (85\%), patrono (80\%), empleado tercerizado (71\%) y privado (66\%). Por su parte, las mujeres presentan mayor concentración en la categoría de empleado doméstico (93\%). La categoría que presenta mayores niveles de equidad 
es la de empleado público, cuya participación es de 56\% de hombres y $44 \%$ mujeres (p. 156) [9].

El trabajo de cuidados y doméstico no remunerado representa la actividad más importante y demandante del tiempo de las mujeres ecuatorianas. La magnitud y naturaleza de este trabajo lo convierte en una tarea fundamental para el bienestar de la población en general, la reproducción social y de la fuerza de trabajo, y el sostenimiento de las familias en los períodos más críticos [10].

No obstante, se trata de un trabajo desvalorizado socialmente, recargado en las mujeres, no retribuido y poco reconocido [11]. Por ello, se constituye en la fuente más importante de pobreza relativa y desventaja de oportunidades de las mujeres, así como en una barrera a la entrada, permanencia y calidad en el mercado laboral.

El análisis desarrollado en Argentina por el Ministerio de Trabajo [12], Empleo y Seguridad Social permite conocer que la tasa de empleo de las mujeres es aproximadamente 25,5 puntos porcentuales menor a los varones, y a escala mundial casi el $40 \%$ de mujeres con trabajos no remunerados con cuentan con seguridad social.

Las tareas a las que se dedican las mujeres son variadas y heterogéneas y, en muchos casos, las realizan sin apoyo adicional familiar y de servicios sociales básicos. Todavía la visión cultural de la población ecuatoriana sobre el cuidado, especialmente de niños/as menores de 5 años, está centrada en el rol tradicional de las madres en la familia. Las políticas y programas del Estado no han buscado cambiar los patrones en este sentido, de manera que se garanticen a las mujeres oportunidades equitativas para su acceso al mercado laboral durante todo su ciclo productivo. Los servicios estatales no llegan a constituirse en un apoyo a la conciliación de la vida laboral y familiar, debido no sólo a problemas de cobertura y calidad, sino también por la ausencia de este enfoque en el diseño de sus programas.

Las responsabilidades del cuidado de hijos/as marcan los ciclos de ingreso y salida del mercado laboral de las mujeres, particularmente de aquellas en situación de pobreza, así como las características marcadamente informales de su inserción laboral. Profundiza, además, las diferencias de oportunidades con mujeres de estratos sociales que cuentan con ingresos para acceder a servicios de cuidado infantil en el mercado, sea acudiendo al trabajo doméstico remunerado o a guarderías particulares [13].

Estudios realizados por la OIT indican:

En los países desarrollados, las mujeres destinan una media diaria de $4 \mathrm{hr}$ y 20 min al trabajo de cuidado no remunerado, y los hombres, 2 hr y 16 min. En los países en desarrollo, las mujeres le dedican $4 \mathrm{hr}$ y 30 min diarios a esta actividad, frente a $1 \mathrm{hr}$ y 20 min de los hombres. Estas diferencias de género se deben, por un lado, a la falta de servicios e infraestructuras que permitan reducir las tareas domésticas y de cuidado no remuneradas, y por otro, a las normas sociales y de género, según las cuales se considera a las mujeres las principales proveedoras de atención primaria (p. 20) [14]. 
La naturalización del rol femenino en los trabajos de cuidado y su ausencia de valor social y económico, permean no sólo las culturas cotidianas de los hogares, sino también las culturas de la gestión pública. Bajo estas características, aún en la oferta pública el trabajo no remunerado de las mujeres pasa a ser un ingrediente principal de los cuidados, a través de modalidades tradicionales de 'participación comunitaria' que no se ha buscado trascender. Por el contrario, la mayor asignación de recursos públicos a la educación en relación a los programas de bienestar social, así como los esfuerzos desplegados para concientizar a la población sobre la importancia de la escolarización de niños/as, evidencia avances importantes hacia una cobertura universal para los grupos de edad de 6 a 11 años [15].

Sin embargo, aún quedan todavía grandes retos en términos de calidad. La participación escolar de niños/as posibilita una redistribución social de los tiempos de cuidado y mayores oportunidades a las mujeres de acceso al mercado laboral, aunque esto no es necesariamente una relación directa por la incompatibilidad de horarios de la jornada escolar y laboral [16].

Por lo general la situación precaria de la mujer, que a más de afrontar con responsabilidad la labor diaria en su trabajo tiene que dedicarse a su casa con muchas actividades las mismas que consisten en: El trabajo de cocina, limpieza, lavado y planchado de ropa, reparaciones y mantenimiento de la vivienda, cuidado de niños/as, cuidado de enfermas/os, coser, tejer o remendar ropa de la familia [17]. A ello debe sumarse también el tiempo invertido en actividades denominadas como auxiliares domésticas en las que se incluye el traslado de miembros del hogar (a la escuela, o a la atención en salud, por ejemplo), y la administración y realización de compras para el hogar.

Con excepción de la actividad de reparaciones y mantenimiento de la vivienda, en todas las demás y con diferente participación de acuerdo a la edad y el parentesco, son las mujeres las que invierten mayor número de horas, aun cuando tengan también un trabajo extra doméstico. Para Espinoza y Gallegos las diferencias son cada vez mas evidentes, 'En el momento que se realiza la entrevista por lo general ya se tiene una idea de las características que debe tener la persona que se debe contratar, marcando una diferencia en que será un hombre o una mujer' (p. 32) [18]. Sin embargo, a pesar de todas las diferencias las mujeres tratan de abrirse caminos.

\subsection{Salario del sector informal}

La dinámica de la economía divide al mercado laboral, encontrándose el sector formal y el sector informal agrupando en el primero a quienes trabajan en empresas registradas y en el sector informal donde se ubican todas aquellas personas que brindan sus servicios en mercados o plazas, quienes se dedican a la agricultura, y quehaceres domésticos entre otras tareas que demandan la práctica de un oficio. En Ecuador la 
lucha porque quienes ejercen estas actividades accedan a una seguridad social apenas comenzó como política de Estado en el 2012, sin embargo según estadísticas del INEC [19], no se reportan incrementos significativos en los empleos no remunerados.

Se puede definir según las Naciones Unidas como sector informal quienes forman parte de los hogares y se constituyen como sociedades, lo establece el mismo documento, el sector informal incluso llega a constituir el $47,5 \%$ del total de personas con empleos.

Como parte de las actividades en las cuales este sector de la economía se desarrolla se puede anotar: El transporte a través de triciclos, restaurantes pequeños conocidos también como fondas, las ventas ambulantes de alimentos preparados en pequeños carros, ventas de otros productos alimenticios no preparados en ese momento sobre todo a la puerta de instituciones de educación o de gran afluencia de personas (aguas, jugos, snacks en funda, etc.), el expendio de productos agrícolas en los mercados.

La presencia de quienes se dedican a esta labor se la puede observar tanto en el sector urbano y rural, evidenciándose por la mayor afluencia de población en las ciudades.

En su mayoría se puede observar que es la mujer la que se dedica a actividades determinadas como el servicio doméstico y la venta ambulante, estos roles se han vuelto parte cultural de la sociedad, haciendo que este fenómeno poco equitativo se vea natural e incluso se acepte como tal por parte de quienes ejercen estas labores adentrándose a las dinámicas de los hogares ecuatorianos las funciones mencionadas [20].

La equidad sin embargo, se asocia el enfoque individual que las agrupaciones sociales iniciando desde la familia pueden tener, puesto que el nivel de familiaridad puede influir en los acuerdos sociales para las funciones que desempeñará cada miembro, el hombre es por naturaleza social y la mayor parte de su vida se desarrolla en compañía de otro ser humano con algún parentesco, estos aspectos son cruciales cuando hablamos de indicadores de género, parámetros de desarrollo social como la autodeterminación, la generación de su propio ingreso, contar con un trabajo, la seguridad y el ámbito social que rodea a un individuo condiciona los recursos que pueden estar a disposición tanto para hombres como para mujeres, sin embargo, una mayor conciencia de un desarrollo individual para un crecimiento colectivo ayuda generar una mejor sinergia de las economías [21].

La diferencia en el salario o ingreso que un individuo puede percibir no solo está condicionada al sector en el cual desarrolla sus actividades es decir, formal e informal, sino también por el género y el rol que le ha sido asignado desde la familia y el medio en el cual se desenvuelve el individuo. 


\subsection{Desarrollo económico social basado en equidad de género}

El desarrollo de una sociedad debe concebirse en una igualdad de género que permita oportunidades tanto a hombres como mujeres de su realización personal, sin embargo, esta condición se ve social y culturalmente condicionada, por tanto se requiere de políticas desde el Estado para modificar conductas a través de la educación.

Tradicionalmente se ha designado tareas como la del trabajo fuera de casa al hombre y a la mujer el cuidado del hogar, los hijos y la familia en tareas que no se consideran remuneradas por la simple asimilación de roles establecidos, limitando el desarrollo de cada individuo de acuerdo al género, en especial de las mujeres, las labores propias del hogar no se consideran como actividades económicas formales o peor aún informales, este es uno de los inconvenientes para las economías puesto que no se regulan dando lugar fácilmente a la explotación silenciosa de talentos y fuerza de trabajo.

La edad se convierte en un factor adicional para establecer las diferencias de género, esta inequidad se visualiza en un mayor porcentaje en jóvenes y adultos, se puede observar mujeres de mayor edad y jóvenes son consideradas para labores informales, aunque se cuente con escaneos públicos de representación social.

América latina evidencia una elevada desigualdad que incide en el desempeño de la economía regional, pero los factores que se engranan para estas realidades no solo pasan por el análisis realizado de la familia como núcleo de la sociedad o los enfoque sociales, se involucran políticas, visiones estratégicas o modelos económicos que incrementan las brechas socio-económicas, quien tiene un mayor ingreso invierte o tiene mayores oportunidades de educación a diferencia de quienes no cuentan con los recursos naciendo otra limitante para quienes no generan su propio ingreso o este es menor como es el caso de las mujeres [22].

\subsection{Puesta en valor de la equidad de género para remuneraciones salariales}

A pesar de las limitaciones la mujer ha intentado ingresar y mantenerse en el mercado laboral, su inserción ha demostrado incrementos significativos, pues su propia concepción como individuo y el desarrollo que debe y puede lograr a cambiado, se ha involucrado en el sector productivo y político ampliando su accionar a otras áreas que en su momento estaban solo asignadas a los hombres, la división sexual del trabajo que es el valor social y económico que se asigna a las tareas que realizan los hombres y las mujeres, la responsabilidad social asumida como el cuidado del hogar y la familia asignada con exclusividad a la mujer [23].

Las estrategias de desarrollo económico permiten la apertura de espacios para la inserción a espacios laborales a través de la promoción del diálogo entre los diferentes 
sectores, sin embargo la tasa de participación femenina sigue siendo inferior, debido a varios factores como la preferencia empresarial hacia los varones, ante ello es necesario que desde las instancias públicas de control y administración socio económica se integre a la empresa, la academia y los gobiernos seccionales para el diseño de una ruta común con intereses para todos y de todos [24].

Por otro lado no solo influye la tasa de ocupación sino la brecha salarial que se ve incrementada con las diferencias sociales, para a OIT [25] en su informe establece que existe un baja capacidad por parte de los países de América Latina para la generación de empleo en el trascurso de los años desde 1990, y aunque al parecer para los hombres parece este indicador que permanece lineal se ve condicionada por edad, siendo más productiva a los 25 años y disminuyendo considerablemente en edad de retiro, el análisis es más profundo para el sector masculino, sin embargo para las mujeres se evidencian las dificultades para conseguir un empleo en periodo mucho más largos que para los varones provocando depresión y desaliento que influye en el nivel de participación.

Algunos países de la región han logrado implementar políticas importantes como las acciones afirmativas en empresas de educación superior, equidad de género para espacios públicos desde la planificación nacional como lo hizo Ecuador en su Plan Nacional de desarrollo 2017-2023, pero los cambios son poco significativos. Para el caso de las mujeres el porcentaje del $38 \%$ como parte de los empleos no formales no ha cambiado, la diferencia se incrementa si se considera el nivel de preparación o con título superior incluso el número de varones en las empresas privadas supera en 2 a uno a espacios de trabajo que pueden tener las mujeres en el mismo sector.

El trabajo se apoya en una investigación de estudios analíticos sobre la incidencia de género y equidad salarial que pueden tener las mujeres de la ciudad de Riobamba en el sector informal, exploratorios que permitan comprender las estrategias que se pueden diseñar enfocadas a un desarrollo equitativo, por otro lado se aplican estudios correlacionales entre las dos variables mencionadas haciendo que esta investigación tenga un enfoque cuantitativo y cualitativo, con metodologías cuantitativas y cualitativas, para ello parte de una revisión bibliográfica y de campo que permita comprender el fenómeno planteado.

Se establece como punto de partida del estudio de las siguientes variables: El género y su incidencia en los salarios de las mujeres que trabajan en el sector informal de la economía en la ciudad de Riobamba, pretende demostrar que el género y el salario son dependientes. Su relación se analiza a través del Chi cuadrado. 


\subsection{Población y muestra}

La proyección de población según el INEC nos permite conocer que para el 2019 la ciudad de Riobamba contó con una población de 261.360 habitantes el cálculo de la muestra es el siguiente:

$$
\begin{gathered}
n=\frac{Z^{2}(p)(q)(N)}{N(e)^{2}+(Z)^{2}(p)(q)} \\
n=\frac{(1,96)^{2}(0,5)(0,5)(764)}{764(0,0301)^{2}+(1,96)^{2}(0,5)(0,5)} \\
N=1056 .
\end{gathered}
$$

Se toma una muestra aleatoria de trabajadores del sector informal para los análisis respectivos.

\section{Resultados y Discusión}

En la ciudad de Riobamba de la muestra estudiada el $80 \%$ de trabajadores informales son mujeres y el 20\% varones de acuerdo a la Figura 2 .

En el sector informal de la provincia de Chimborazo, las mujeres están sobre representadas en especial en los sectores derivables de la agricultura, o lo que en otras palabras se puede señalar, existe una brecha de formalidad entre hombres y mujeres, esto coadyubado por el dato entregado por el INEC, en el cual se señala que un total de 2,7 millones hacen de su sustento diario la informalidad [26].

\section{Table 1}

Resultado de frecuencia de género.

\begin{tabular}{l|ll} 
Genero & Frecuencia & Porcentaje \\
\hline Femenino & 822 & 77,8 \\
\hline Masculino & 234 & 22,2 \\
\hline Total & 1056 & 100
\end{tabular}

Del $77,8 \%$ de mujeres que trabajan en el sector informal el 78,2\% proviene del sector rural, donde las diferencias de género son mucho mas arraigadas culturalmente en desventaja para las mujeres según la Figura 2.

La presencia mayoritaria de la mujer en el sector informal ecuatoriano, obedece a una gestión no transparente en el país, junto con una serie de desaciertos institucionales, estatales e inclusive culturales que han llevado a que la mujer tome el rol de manutención en una gran parte de los hogares ecuatorianos. 


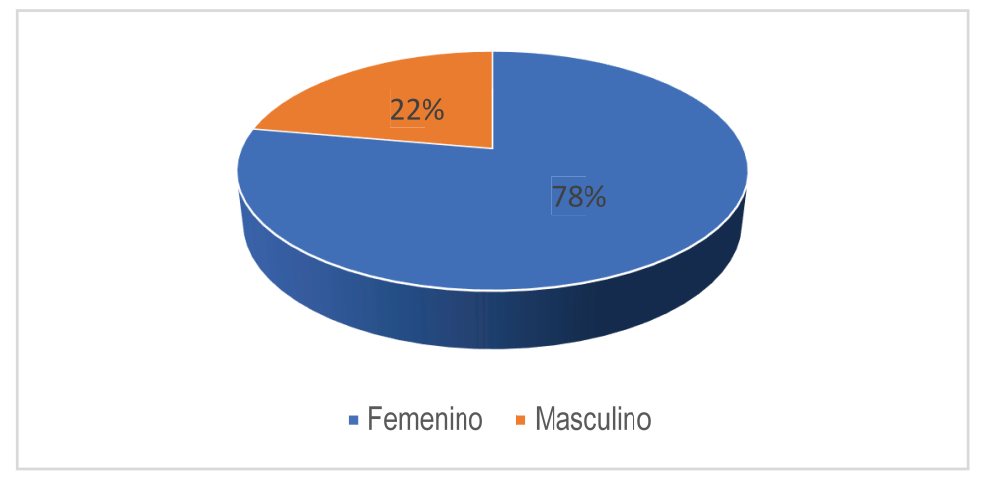

Figure 1

Presencia de la mujer en el sector informal de la economía.

\section{Table 2}

Lugar de procedencia de las mujeres que trabajan en el sector informal.

Sector de origen
Urbano
Rural
Total

Frecuencia
179
643
822

Porcentaje

21,8

78,2

100

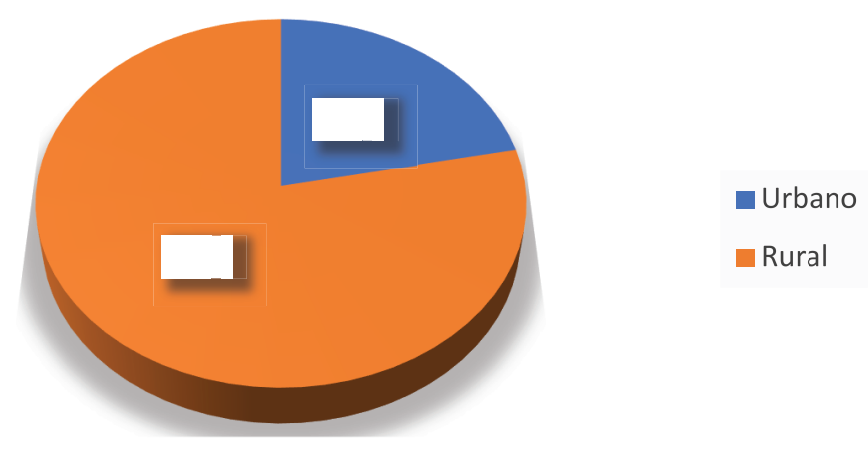

\section{Figure 2}

Lugar de procedencia de las mujeres que trabajan en el sector informal.

Se pudo identificar además que el $43 \%$ de las mujeres que trabajan en el sector informal son jóvenes, seguido por el 31,8\% que son adultos entre los datos más significativos, sin embargo, es importante anotar la presencia de niñas en $14,4 \%$.

Las tareas de cuidado del hogar no es la única función de mujer en el sector informal, el $92 \%$ se identifica como jefe de familia, con un promedio de personas a su cuidado que va de 3 a 5 .

El factor salarial en el sector informal obdece a una concepcion de desavalorizacion del trabajo por parte de los diferentes actores sociales, del acual ahupado de una deficiente gestion gubernamental y poco control municipal hace de este sector un trabajo de reconocimiento salarial sumamente deficiente, el $66 \%$ de mujeres quw 
Table 3

Porcentaje de mujeres por grupos etarios que trabajan en el sector informal.

\begin{tabular}{l|ll} 
Grupos etarios & Frecuencia & Porcentaje \\
\hline Niñas & 118 & 14,4 \\
\hline Jóvenes & 56 & 43,3 \\
\hline Adultos & 261 & 31,8 \\
\hline Tercera edad & 87 & 10,6 \\
\hline Total & 822 & 100,0
\end{tabular}

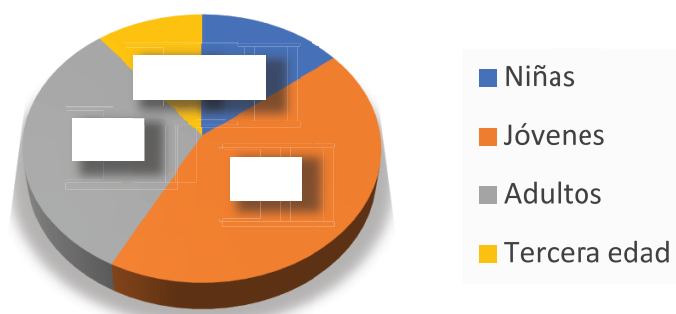

\section{Figure 3}

Porcentaje de mujeres por grupos etarios que trabajan en el sector informal.

Table 4

Condición de mujer como cabeza de hogar.

\begin{tabular}{l|l|l} 
Condición como cabeza de hogar & Frecuencia & Porcentaje \\
\hline Si & 759 & 92,3 \\
No & 63 & 7,7 \\
Total & 822 & 100,0
\end{tabular}

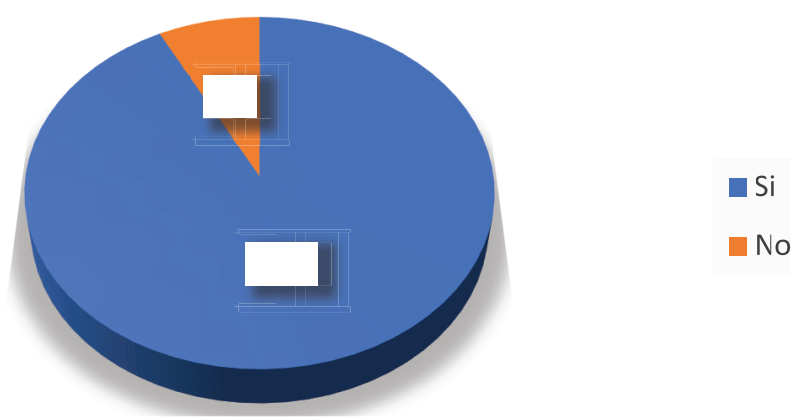

\section{Figure 4}

Condición de mujer como cabeza de hogar.

trabaja en el sector informal gana de $\$ 100$ a $\$ 400$ mensuales con un promedio de $\$ 263$.

Las oportunidades laborales representadas por el salario y el género de trabajadores informales del sector urbano de la ciudad de Riobamba son dependientes. 


\section{Table 5}

Tabla del salario mensual hombres.

\begin{tabular}{lll} 
Salarios & Frecuencia & Porcentaje \\
\hline Menor a 100 & 2 & 0,19 \\
100 a 400 & 697 & 66,00 \\
\hline 401 a 800 & 319 & 30,21 \\
Mas de 800 & 38 & 3,60 \\
\hline Total & 1056 & 100,00
\end{tabular}

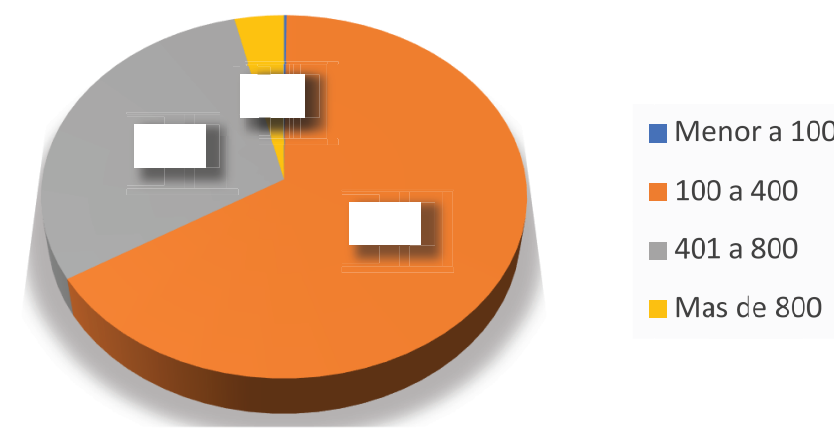

Figure 5

Salario de las mujeres.

\section{Table 6}

Tabla de contingencia salarios de acuerdo a género en la ciudad de Riobamba.

\begin{tabular}{ll|l|l|l|l} 
Salarios & $\begin{array}{l}\text { Género } \\
\text { Masculino }\end{array}$ & Fe & Femenino & Fe & Total \\
\hline Menor a 100 & 0 & 3,28 & 2 & 11,54 & 4 \\
\hline 100 a 400 & 166 & 55,83 & 531 & 196,13 & 68 \\
\hline 401 a 800 & 41 & 142,86 & 278 & 501,85 & 174 \\
\hline Mas de 800 & 27 & 32,02 & 11 & 112,48 & 39 \\
\hline Total & 234 & & 822 & & 285
\end{tabular}

$\mathbf{X}^{2} \mathbf{O}$

El género y el pago salarial de los trabajadores informales son independientes 


\section{$\mathbf{X}^{2} \mathbf{a}$}

El género y el pago salarial de los trabajadores informales no son independientes

$\mathbf{X}^{2}$ 。

$$
\begin{aligned}
& =\sum_{i=1}^{k}\left(\frac{(O i-E i)(O i-E i)}{E i}\right) \\
& =\sum_{i=1}^{k}\left(\frac{(234-4)(234-4)}{4}\right)
\end{aligned}
$$

$X^{2}{ }^{2}=1065,20$

Valor crítico de $a=0,05$

$\mathbf{X}_{\mathrm{a}}{ }_{\mathrm{a}}=(r-1)(c-1)$

$\mathbf{X}^{2}{ }_{a}=7,8$

En base a los resultados obtenidos se determina que los salarios están en constante dependencia del género de los trabajadores informales como se muestra en las Tablas 1 y 2.

Para la Oficina Regional de la OIT [23] el 53,7\% del mercado laboral femenino, trabaja en condiciones de informalidad, esto a pesar del incremento del sector femenino al mercado laboral formal del país, a pesar de su contribución como generadora de recursos en su hogar, su mayor nivel educacional, junto con su cada vez mayor aporte en la manutención, se mantienen invariables patrones de desigualdad de género no solo en Ecuador sino también en América Latina.

En el Ecuador antiguo (siglo XIX), se tenía la percepción del trabajo y la distribución y responsabilidad de los roles en el hogar, correspondiéndoles a las mujeres su participación total en el mismo a través del cuidado y mantenimiento de los hijos, así como de proveedora de alimento, del cual con los años y costumbres propias del indígena ecuatoriano se traslado a las grandes urbes como efecto de la industrialización, de la cual hasta el momento esta costumbre se ha mantenido, mostrando como sociedad una deficiente evolución de pensamiento y convivencia social.

Estrategia para promover la equidad en el reconocimiento y puesta en valor del trabajo equitativo sin distinción de género:

La incorporación del enfoque de género en las políticas públicas requiere la voluntad política de institucionalizar planes, acuerdos, medidas y mecanismos dirigidos a 


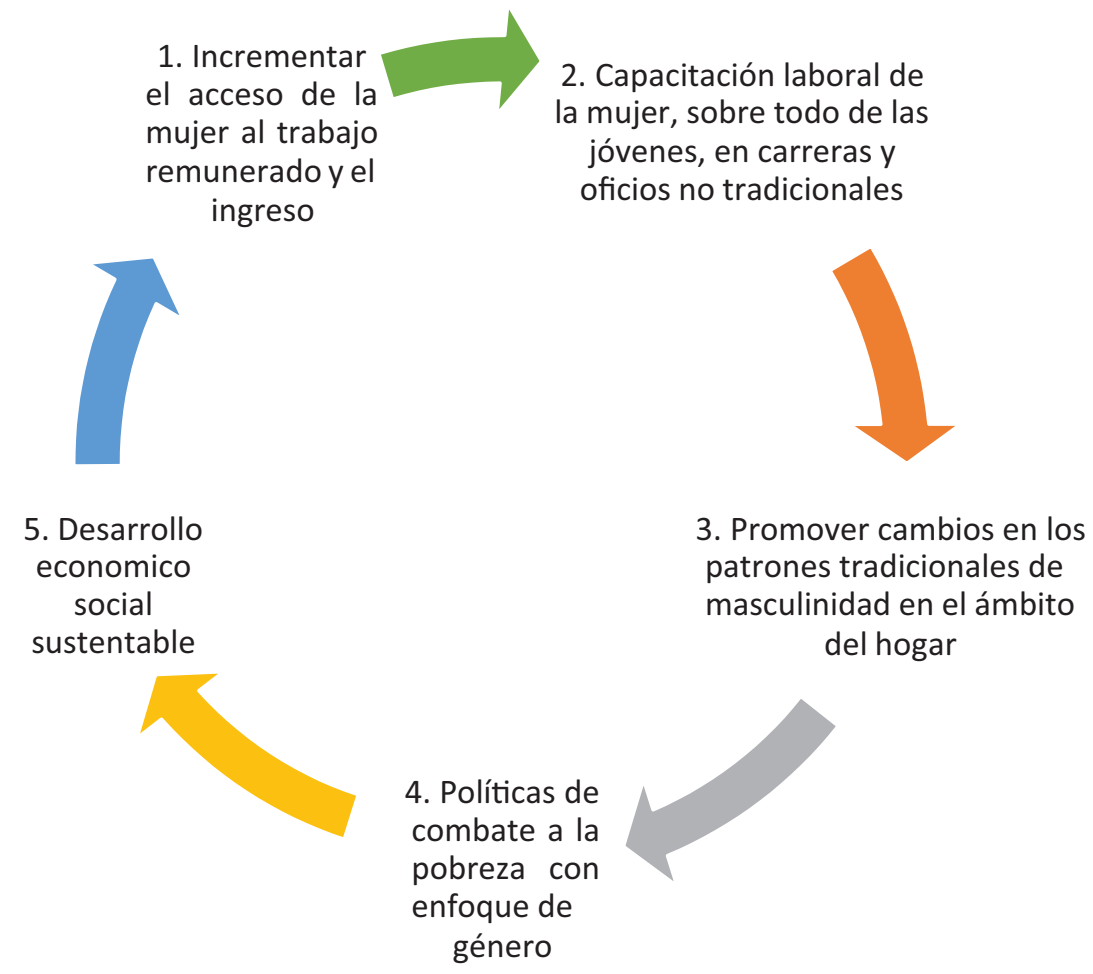

Figure 6

Estrategia para promover la equidad en el reconocimiento y puesta en valor del trabajo equitativo sin distinción de género.

disminuir progresivamente la desigualdad entre hombres y mujeres, como se muestra en la Figura 6.

Para lograr esto, es imprescindible continuar incrementando la inversión social al tiempo que se intensifica y cualifica la labor que realiza el Estado en favor de la Mujer de sensibilizar y capacitar en el enfoque de género al personal directivo y técnico de todas las instancias públicas [27]. Es importante enfatizar la importancia de que estas acciones incluyan las instituciones públicas responsables de las políticas económicas y las culturales.

Al incidir en el quehacer de las instituciones vinculadas a la cultura se fomentan cambios necesarios en los patrones tradicionales de masculinidad tanto en el ámbito de la familia, como de los espacios laborales y de poder político [28].

Dada su complejidad y su naturaleza estructural, la problemática de género debe ser abordada de forma integral, con medidas de gran impacto en términos de cobertura poblacional y de su poder de incidencia en la cultura y la economía [29]. En ese sentido, creo que lo que falta en nuestro país, no son tantas propuestas técnicas novedosas y viables, pues ya han sido planteadas en documentos, sino la voluntad política expresada en apoyo financiero para ejecutarlas.

Incrementar el acceso de la mujer al trabajo remunerado y el ingreso [30]: 
1. Exenciones impositivas y reconocimientos a las empresas privadas cuyo personal femenino constituya más del $60 \%$.

2. Exenciones impositivas y reconocimientos a las empresas que establezcan estancias infantiles.

3. Exenciones impositivas y reconocimientos a las empresas privadas que desarrollen programas sociales tendentes a incrementar el salario indirecto (bonos para adquisición de vivienda, becas, bonos para transporte, planes vacacionales, etc.).

4. Crédito para mujeres a tasas preferenciales.

Capacitación laboral de la mujer, sobre todo de las jóvenes, en carreras y oficios no tradicionales:

1. Cuotas femeninas progresivas hasta alcanzar el $50 \%$ de la matrícula de los centros de capacitación técnico- profesional y universidades en las áreas y carreras en las que la mujer es minoritaria.

2. Reconocimientos a las mujeres egresadas de esos centros técnicos y universitarios con las mejores calificaciones y garantía de empleo bien remunerado.

\section{Promover cambios en los patrones tradicionales de masculinidad en el ámbito del} hogar:

1. Campañas permanentes y masivas a través de la escuela, medios de comunicación y organizaciones comunitarias estimulando la división equitativa del trabajo doméstico.

\section{Políticas de combate a la pobreza con enfoque de género:}

1. Focalización en los segmentos de población femenina más vulnerables tales como: discapacitadas, tercera edad, trabajadoras domésticas, trabadoras sexuales, mujeres domínico-haitianas, inmigrantes haitianas, jóvenes rurales, emigradas al exterior y jefas de hogar desempleadas.

2. Revisión de los presupuestos dedicados al combate a la pobreza para adecuarlos como herramienta de reducción de la desigualdad de género.

\section{Desarrollo económico social sustentable:}

Las condiciones imprescindibles para el éxito de las estrategias propuestas son un incremento sostenido del gasto público social, la existencia de un sistema eficiente de monitoreo y evaluación de las políticas públicas y la articulación efectiva entre Estado, ONG, agencias de cooperación internacional y organizaciones comunitarias [31]. 
Ciertamente estamos hablando de revolucionar las costumbres seculares, las prácticas políticas, los valores tradicionales, los patrones de relacionamiento entre los seres humanos para lo cual es imprescindible que mujeres y hombres con conciencia de género asuman posiciones de poder en el sector público y en el privado, mujeres y hombres que reconozcan que para lograr la igualdad de género y el desempeño pleno de la ciudadanía de las mujeres es necesario tomar medidas afirmativas.

Estamos soñando con erradicar la guerra puertas adentro que mata más de un centenar de mujeres al año, dejando centenares de huérfanos, con detener el autoexilio que se lleva cientos de miles de mujeres a Europa y Estados Unidos, fragmentando para siempre las familias, con evitar los miles de nacimientos no deseados que tronchan las aspiraciones de nuestras adolescentes, en fin, con quesea compatible el desarrollo intelectual, la estabilidad emocional y la evolución espiritual de los hombres y de las mujeres de nuestro país. Ciertamente lograr todo esto es tan difícil como aparenta, pero posible y sobre todo, justo.

Como nos hemos podido dar cuenta esta dura realidad deben conllevar un alto grado de incentivo para que todos los organismos del estado asuman políticas y objetivos estratégicos que deberán ser definidos en un Plan de Igualdad de Oportunidades, declarando como política de Estado, no se puede admitir disparidad e inequidad de género, esto solo si buscamos un sociedad justa y con equidad, entonces primero se deberá:

1. Identificar las políticas públicas de género, y construir metas que en el mediano y largo plazo determinen los cambios y logros alcanzados por el país, en su objetivo por llegar a la igualdad plena entre hombres y mujeres.

2. Se debe invertir en la educación de niñas y mujeres que son las que contribuye de manera importante a la superación de la pobreza.

3. Incrementos salariales en trabajos tradicionalmente femeninos o de bajos ingresos.

4. Evaluar puestos de trabajo orientados a luchar contra la infravaloración salarial de las mujeres.

5. Iniciativa de realizar comparaciones salariales del sector público y privado.

6. Brindar oportunidades de trabajo a igual preparación igual valor.

7. Crear más centros de cuidado infantil con condiciones favorables para el crecimiento y formación de los niños y niñas menores de edad.

8. Políticas educativas donde se aniquile el machismo y la inequidad. 
9. La contratación y retención de profesionales que den atención socio sanitaria que debe ir desde la mejora salarial junto a la progresión de la carrera profesional, y condiciones de trabajo de calidad, procurando incluir equidad de género.

10. Debe ponerse una base de datos sobre indicadores de brechas salariales de género como servicio asistencial y continuo.

11. Jornada laboral diferente al hombre por su responsabilidad del hogar, cuando hay niños de corta edad.

\section{Conclusiones}

La diferencia de género tiene una incidencia directa en el salario percibido por la mujer, a quienes, a más de su responsabilidad de rol dentro del núcleo familiar, se le ha impuesto por dogma de vida su papel; de sostén de hogar y en muchos casos de manutención. En la ciudad de Riobamba de la muestra estudiada el $80 \%$ de trabajadores informales son mujeres y el $20 \%$ varones lo que evidencia una brecha muy marcada que no es evidenciada en datos nacionales, puesto que se cuentan con estadísticas mayoritariamente del sector formal de la economía cuando este es uno de los sectores más amplios, puesto que se ha convertido en la fuente de autoempleo para las familias de clase media baja y baja, y más aún del sector rural que migra a las ciudades, dentro del estudio se identificó que el $78,2 \%$ de mujeres provienen del sector rural, bajo estas condiciones la mujer asume la responsabilidad de conseguir el sustento económico y cuidado familiar por las concepciones culturales que no han permitido que evolucionen los roles determinados socialmente, otra de las características que se evidencias es la presencia del trabajo infantil de niñas en un $14,4 \%$ de la población femenina, el salario para las mujeres en un $66 \%$ va de 100 a 400 mensuales y un promedio de $\$ 263$ que no le permite cubrir sus necesidades básicas, siendo que el $92,3 \%$ se identifica como jefe de hogar y tiene a su cargo de 3 a 5 personas bajo su cuidado en promedio.

Transformando este tipo de problemática social, en un tema a considerar dentro de la política gubernamental, a fin de dictaminar estrategias socioeconómicas con medidas que tengan como objetivo una verdadera equidad, debiendo establecer formas de censo más inclusivas ya que esta actividad económica se la realiza hasta en forma clandestina debido a la serie de trámites burocráticos que afronta el ingreso a estos centros de comercialización.

Así se plantea la 'Estrategia para promover la equidad en el reconocimiento y puesta en valor del trabajo equitativo sin distinción de género', basada en cinco componentes para promover: (i) La implementación de verdaderas políticas de inclusión y de control; (ii) La capacitación de la mujer, sobre todo de las jóvenes, en carreras y oficios no tradicionales; (iii) Promover cambios en los patrones tradicionales de masculinidad en 
el hogar; (iv) Políticas de combate a la pobreza con enfoque de género que promuevan como punto; (v)Desarrollo económico social sustentable que considere las características propias de cada espacio geográfico y sectores productivos como el informal donde encontramos brechas y problemas socio económicos más profundos.

\section{References}

[1] Organización de las Naciones Unidas. Igualdad de género, Porqué es importante. ONU; 2015.

[2] OIT. Iniciativa relativa a las mujeres en el trabajo: impulso en favor de la igualdad. Ginebra: Organización MUndial del Trabajo; 2018.

[3] Instituto Nacional de Estadísticas y Censos. Encuesta Nacional de Empleo, Desempleo y Subempleo (EMENDU). Quito: INEC; 2019.

[4] GAD Municipal del cantón Riobamba. Plan de desarrollo y ordenamiento territorial 2015 - 2019. Riobamba: GAD Riobamba; 2015.

[5] Melendres E. Brechas Salariales por género en la ciudad de Riobamba. Riobamba: Española; 2014.

[6] Lechuga J, Ramirez G, Guerrero M. Educación y género. El largo trayecto de la mujer hacia la modernidad en México. 2018;15(43),110.

[7] Gomez VJ. Corresponsabilidad familiar y el equilibrio trabajo-familia: medios para mejorar la equidad de género. Polis, Revista Latinoamericana. 2015.

[8] Rodríguez R, Limas M. El análisis de las diferencias salariales y discriminación por género por áreas profesionales en México, abordado desde un enfoque regional, 2015. Estudios Sociales; 2017.

[9] Castillo J, Salas C. Estabilidad laboral y desigualdad del ingreso: una perspectiva de género. Cuestiones Económicas. 2018;28:149.

[10] Vaca I. Oportunidades y desafíos para la autonomía de las mujeres en el futuro escenario del trabajo. Serie asuntos de género CEPAL; 2019.

[11] OIT. Informe Mundial sobre Salarios 2018/2019, Que hay detrás de la brecha salarial de género. Ginebra: Organización Mundial del Trabajo; 2018.

[12] Ministerio de Trabajo. Las mujeres en el mundo del trabajo. Argentina: Ministerio de Trabajo; 2017.

[13] OIT. Hacia un futuro mejor para las mujeres en el trabajo: La opinión de las mujeres y de los hombres. Organización Mundial del Trabajo; 2017.

[14] OIT. Las mujeres en el trabajo. Tendencias de 2016. Oficina Internacional del Trabajo; 2016.

[15] FAO. Manual y caja de herramientas de perspectiva. Mexico: Organización de las Naciones Unidas para la Alimentación y la Agricultura; 2018. 
[16] PNUD. Desarrollo económico local y género: Una agenda para un desarrollo local más igualitario e. Centro Regional para América Latina y el Caribe; 2017.

[17] Hernández M, Ibarra L. Conciliación de la vida familiar y laboral. Un reto para México. Iztapalapa Revista de Ciencias Sociales y Humanidades. 2019;(86):159-184.

[18] Espinoza M, Gallegos D. Discriminación laboral en el Ecuador. Espacios. 2018;39(23):32.

[19] INEC. Encuesta Nacional de Empleo, desempleo y Subempleo - ENEMDU. Quito: INEC; 2018.

[20] Mayorga, K. Igualdad de género en la educación Superior en el siglo XXI. Palermo Business Review. 2018;137-144.

[21] CEPAL. Asuntos de género. La Agenda 2030 y la Agenda Regional de Género. Santiago: Naciones Unidas; 2017.

[22] Cortés A, Flores M. Diferencias salariales por género en el departamento de Santander, Colombia. 2016;35(61).

[23] Oficina Internacional del Trabajo. (2018). Perspectivas sociales y del empleo en el mundo mujeres, Tendencias del empleo femenino 2018 Agenta global. Ginabra: Oficina Internacional del Trabajo; 2018.

[24] Campaña M, Melendes E, Flores J, Acosta R. Administración de empresas de economía social y solidaria. MKT, Descubre; 2019.

[25] OIT. Panorama Laboral 2019 América Latina y el Caribe. Lima: Organización Internacional del Trabajo; 2019.

[26] Lideres R. La informalidad laboral, una condición arraigada en Ecuador. LIDERES; 2015.

[27] FMI. Las mujeres y el crecimiento económico. Finanzas y desarrollo. 2019;12.

[28] AECID. Género y Desarrollo. Más de una década de cooperación española por los derechos de las mujeres en Ecuador. Ecuador: Edición Agencia Española de Cooperación Internacional para el Desarrollo (AECID); 2017.

[29] García M, Fatou B. Economía, Género y desarrollo enfoques e iniciativas hacia la igualdad. Madrid: Universidad Complutense de Madrid, 2016.

[30] CEPAL. Planes de igualdad de género en América Latina y el Caribe Mapas de ruta para el desarrollo. Santiago: Naciones Unidas, CEPAL; 2019.

[31] Flores J, Campaña M, Melendres E, Acosta R. Proceso administrativo, contable en los negocios y la competitividad para la gestión económica local. MKT, Descubre; 2019. 